\title{
Qualidade físico-hídrica e rendimento de soja (Glycine max L.) e feijão (Phaseolus vulgaris L.) de um Argissolo Vermelho distrófico sob diferentes sistemas de manejo
}

\author{
Physical-hidric quality and soybean (Glycine max L.) and black bean (Phaseolus vulgaris L.) \\ yield of a Rhodic Paleudalf under different tillage systems
}

\author{
Cláudia Liane Rodrigues de Lima ${ }^{1}$ Dalvan Jose Reinert ${ }^{2}$ José Miguel Reichert ${ }^{2}$ \\ Luis Eduardo Akiyoshi Sanches Suzuki ${ }^{3}$ Paulo Ivonir Gubiani ${ }^{4}$
}

\section{RESUMO}

A qualidade físico-hídrica tem sido alterada pelo processo de compactação a que os solos estão sendo submetidos nos diversos sistemas de produção agrícola. Este estudo objetivou avaliar a qualidade físico-hídrica de um Argissolo Vermelho distrófico submetido a diferentes manejos. Um experimento com a cultura da soja e dois com a cultura do feijoeiro foram instalados em um delineamento inteiramente casualizado. No ano agrícola 2004/2005, foram testados sete níveis de compactação: $S D$ = sistema de semeadura direta desde o ano de 1989; $S D_{E S C 1}=$ sistema de semeadura direta escarificado em dezembro de 2004; $S D_{E S C 2}=$ sistema de semeadura direta escarificado em dezembro de 2002 e fevereiro de 2004; $S D_{\text {Сомр1 }}=$ sistema de semeadura direta com quatro passadas de máquina de $10 \mathrm{Mg}$ em dezembro de 2001/2002; $S D_{\text {СомР2 }}=$ sistema de semeadura direta com quatro passadas de máquina de $10 \mathrm{Mg}$ em dezembro de 2002/2003; $S D_{\text {СомР3 }}=$ sistema de semeadura direta com quatro passadas de máquina de $10 \mathrm{Mg}$ em dezembro de 2001/2002 e 2002/2003 e SC = sistema convencional de preparo do solo. As maiores alterações na qualidade físico-hídrica foram observadas no sistema convencional de preparo e semeadura direta que recebeu compactação adicional em dois anos agrícolas $\left(S D_{\text {СомРз }}\right)$. O SD apresentou as melhores condições físico-hídricas e permaneceu por maior período dentro da faixa de umidade volumétrica considerada ótima para o desenvolvimento das culturas. A condutividade hidráulica de solo saturado não apresentou diferença entre os tratamentos. O rendimento da soja não foi influenciado pelos tratamentos, enquanto que a escarificação $\left(S D_{E S C 1}\right)$ foi favorável ao rendimento do feijoeiro.

Palavras-chave: condutividade hidráulica do solo, densidade do solo, porosidade do solo, umidade volumétrica, compactação do solo.

\begin{abstract}
The physical-hidric quality has been affected by the compaction process that soils are being undergone in different agricultural systems. The objective of this study was to evaluate the physical-hydric quality of a Rhodic Paleudalf undergone to different tillages. One experiment with soybean and two with black bean were installed using a completely randomized experimental design. Seven compaction levels were tested in the agricultural year of 2004/2005: NT= no tillage since 1989; $N T_{\text {PLOW1 }}=$ no tillage that received a chisel plough in december of 2004; $N T_{\text {PLOW2 }}=$ no tillage that received a chisel plough in december of 2002 and february of 2004; $N T_{\text {СОМР1 }}=$ no tillage with four passes of a buck loader of $10 \mathrm{Mg}$ in december of the 2001/2002; $N T_{\text {СОмP2 }}=$ no tillage with four passes of a buck loader of $10 \mathrm{Mg}$ in december of 2002/2003; $N T_{\text {СОМР3 }}=$ no tillage with four passes of a buck loader of $10 \mathrm{Mg}$ in december of the agricultural year 2001/2002 and 2002/2003 and CT = conventional tillage. The largest changes in the physical-hidric quality were observed in the conventional and no tillage that received additional compaction in two agricultural years $\left(N T_{\text {СомР3 }}\right)$. No tillage (NT) presented the best physical-hidric conditions of soil and stayed longer within the volumetric moisture range considered ideal for crop development. The hydraulic conductivity of saturated soil did not differ among the treatments. Soybean yield was not affected by the treatments, whereas the chisel plough $\left(N T_{P L O W 1}\right)$ was favorable to the black bean yield.
\end{abstract}

Key words: soil hydraulic conductivity, bulk density, soil porosity, volumetric moisture, soil compaction.

\footnotetext{
${ }^{1}$ Departamento de Solos, Universidade Federal de Santa Maria (UFSM). Estrada RS 509, Ed. Zimermann, n.7300, apto. 21, Bairro Camobi, 97110-620, Santa Maria, RS, Brasil. E-mail: clrlima@yahoo.com.br. Autor para correspondência.

${ }^{2}$ Departamento de Solos, UFSM, Santa Maria, RS, Brasil.

${ }^{3}$ Programa de Pós-graduação do Curso de Engenharia Florestal, UFSM, Santa Maria, RS, Brasil.

${ }^{4}$ Curso de Agronomia, UFSM, Santa Maria, RS, Brasil.
} 


\section{INTRODUÇÃO}

O interesse em avaliar a qualidade do solo tem aumentado por considerá-lo como um componente fundamental na manutenção e na sustentabilidade dos sistemas de produção agrícola. Apesar de vários fatores dificultarem em termos quantitativos, sua definição tem sido indicada como a capacidade do solo em promover ao sistema radicular condições adequadas ao crescimento e à produtividade das culturas (WU et al., 2003; KARLEN et al., 2003).

A estrutura de um solo ideal é aquela que permite uma adequada área de contato entre as raízes e o solo, um espaço poroso contínuo e suficiente para o movimento de água e de gases e resistência do solo à penetração que não limite o crescimento de raízes e folhas (KOPPI \& DOUGLAS, 1991).

Estratégias têm sido desenvolvidas na obtenção do incremento da produtividade agrícola associadas à manutenção da qualidade do solo. No entanto, a compactação tem se destacado em nível mundial como um dos principais responsáveis pela degradação da qualidade físico-hídrica de solos submetidos a diferentes sistemas de manejo e pela redução da produtividade das culturas.

Fatores externos e internos condicionam a resposta do solo à compactação e, em decorrência disso, o grau de degradação da qualidade estrutural. Os fatores externos são caracterizados pelo tipo, intensidade e freqüência da pressão exercida por máquinas agrícolas, pelos equipamentos de transporte ou pelo pisoteio de animais e pela compactação atual do solo; enquanto os internos, pelas propriedades físicas, mais particularmente, pela textura e pela umidade do solo, e pelo teor de carbono orgânico (SILVA et al., 2000; DEFOSSEZ \& RICHARDS, 2002). Acompactação, ao causar modificações na estrutura do solo, pode limitar a adsorção e a absorção de nutrientes e, por sua vez, resultar em problemas no estabelecimento e no crescimento de raízes. Essa limitação é originada por alterações em parâmetros físicos como densidade, resistência à penetração e porosidade do solo (LHOTSKÝ et al., 1991; FLOWERS \& LAL, 1998).
A condutividade hidráulica, apesar de ser um indicador variável, pode também revelar diferenças entre sistemas de manejo (COQUET et al., 2005). O incremento da pressão aplicada por máquinas agrícolas pode reduzir os valores de condutividade hidráulica de solo saturado (HORN et al., 2003), corroborando os resultados de OSUNBITAN et al. (2005).

Vários estudos têm relacionado níveis de compactação, propriedades do solo e crescimento das plantas, em sistema de semeadura direta (STONE \& SILVEIRA, 1999; SECCO et al., 2004; COLLARES, 2005). No entanto, ainda existem dúvidas sobre a intensidade de compactação na qual sistemas de manejo possam influenciar negativamente os atributos físico-hídricos do solo, o crescimento e o rendimento das culturas.

O objetivo desse estudo foi avaliar a qualidade físico-hídrica de um Argissolo Vermelho distrófico sob diferentes manejos, a partir da condutividade hidráulica, da densidade, da porosidade total, da macro e microporosidade, da variação temporal da umidade volumétrica do solo, da faixa de umidade ótima e do rendimento de soja e feijoeiro.

\section{MATERIAL E MÉTODOS}

Três experimentos, um com a cultura da soja (Glycine $\max$ L.) e dois com a cultura do feijoeiro (Phaseolus vulgaris L.), em um delineamento inteiramente casualizado, compreendendo 36 parcelas de $6 \times 7$ m e sete tratamentos (Tabela 1 ), foram instalados numa área experimental do Departamento de Solos da Universidade Federal de Santa Maria, RS (29 41' S; $53^{\circ}$ 48” W; 95m).

O clima da região é caracterizado como Cfa (subtropical úmido sem estiagens), conforme classificação de Köppen, sendo a temperatura média do mês mais quente superior a $22^{\circ} \mathrm{C}$ e a do mês mais frio entre $-3^{\circ} \mathrm{C}$ e $18^{\circ} \mathrm{C}$. O solo foi classificado como Argissolo Vermelho distrófico arênico (EMBRAPA, 1999), pertencente à unidade de mapeamento São Pedro (DALMOLIN et al., 2004).

Tabela 1 - Especificação dos tratamentos utilizados.

\begin{tabular}{ll}
\hline Tratamentos & Especificação \\
\hline SD & Semeadura direta desde o ano de 1989 \\
SD $_{\text {ESC1 }}$ & Semeadura direta escarificado em dezembro de 2004 \\
SD $_{\text {ESC2 }}$ & Semeadura direta escarificado em dezembro de 2002 e fevereiro de 2004 \\
SD $_{\text {COMP1 }}$ & Semeadura direta com 4 passadas de máquina de $10 \mathrm{Mg}$ em dezembro de 2001/2002 \\
SD $_{\text {COMP2 }}$ & Semeadura direta com 4 passadas de máquina de $10 \mathrm{Mg}$ em dezembro de 2002/2003 \\
SD $_{\text {COMP3 }}$ & Semeadura direta com 4 passadas de máquina de $10 \mathrm{Mg}$ em dezembro de 2001/2002 e 2002/2003 \\
& Sistema convencional de preparo do solo (uma aração e duas gradagens) \\
\hline
\end{tabular}


A semeadura das culturas foi realizada em dezembro de 2004 e, após um mês, em cada parcela foi coletada uma amostra com estrutura de solo preservada nas camadas de $0-0,05 \mathrm{~m} ; 0,10-0,15 \mathrm{~m} ; 0,20-0,25 \mathrm{~m}$ e 0,30 0,35m, utilizando cilindros metálicos, de diâmetro interno de $0,06 \mathrm{~m}$ e altura de $0,05 \mathrm{~m}$. As amostras foram utilizadas na determinação da condutividade hidráulica de solo saturado $\left(\mathrm{K}_{\theta \mathrm{S}}\right)$ (três repetições), com o auxílio de um permeâmetro de carga constante, da densidade do solo (D $)$ (BLAKE \& HARTGE, 1986), da porosidade total $\left(\mathrm{P}_{\mathrm{T}}\right)$, da macro $\left(\mathrm{M}_{\mathrm{A}}\right)$ e da microporosidade $\left(\mathrm{M}_{\mathrm{I}}\right)$ pelo método da mesa de tensão (EMBRAPA, 1997). Os resultados obtidos foram analisados por contrastes ortogonais a 5\% de significância.

Nos experimentos com o cultivo do feijoeiro, por um período de aproximadamente 60 dias após a emergência das culturas (DAE), foi feito o estudo temporal da umidade volumétrica do solo $(\theta)$, utilizandose um TDR-100 conectado a um medidor e armazenador de dados e hastes metálicas inseridas horizontalmente ao solo nas profundidades de 0,05 e 0,15m.

A partir de equações de limite inferior $\left(R P=24,9929 \times \theta^{-1,0896} \times D_{S}^{4,9261}\right)$ e superior $\left(\theta=0,1387+0,0492 \times D_{s}\right)$ de umidade ótima para esse solo (COLLARES, 2005) e de resistência do solo à penetração (RP) de $2 \mathrm{MPa}$, avaliou-se a faixa de umidade ótima.

\section{RESULTADOS E DISCUSSÃO}

O maior e o menor valor médio $\mathrm{K}_{\theta \mathrm{S}}$ e $\mathrm{D}_{\mathrm{S}}$ foram obtidos respectivamente no $\mathrm{SD}$ e $\mathrm{SD}_{\text {СОмР3 }}$ e no $\mathrm{SD}_{\text {СОмР3 }}$ e SC. Um menor valor médio de $\mathrm{K}_{\theta \mathrm{S}}\left(10,42 \mathrm{~mm} \mathrm{~h}^{-1}\right)$ esteve associado a uma maior $\mathrm{D}_{\mathrm{s}}\left(1,82 \mathrm{mg} \mathrm{m}^{-3}\right)\left(\mathrm{SD}_{\mathrm{COMP} 3}\right)$ (Tabela 2). No processo de compactação, os poros maiores, responsáveis pela aeração do solo, tendem a diminuir, sendo substituídos por poros menores (BOONE \& VEEN, 1994), diminuindo por sua vez a $\mathrm{K}_{\theta \mathrm{s}}$. OSUNBITAN et al. (2005) revelam que a porosidade total do solo pode não ser o principal determinante da condutividade hidráulica do solo.

Diferenças nos valores médios de $\mathrm{P}_{\mathrm{T}}, \mathrm{M}_{\mathrm{A}} \mathrm{e}$ $M_{I}$ podem estar relacionadas à compactação adicional e às operações de aração e gradagem realizadas na área (Tabela 2).

Analisando-se os contrastes ortogonais, nenhum efeito significativo foi observado para a $\mathrm{K}_{\theta \mathrm{S}}$ em todas as profundidades (Tabelas 3 e 4). O elevado intervalo de variação desse parâmetro $(111,50 \leq \mathrm{CV} \leq$ $247,91 \%$ ) influenciou os resultados obtidos. De acordo com WARRICH \& NIELSEN (1980), o coeficiente de variação da $\mathrm{K}_{\theta \mathrm{S}}$ poderá atingir valores maiores do que $420 \%$. Devido à grande variabilidade, GUROVICH (1982) afirmou que é comum não se encontrarem diferenças significativas entre os tratamentos, corroborando resultados de LAL et al. (1999). Sugerese, portanto, que esse parâmetro seja analisado juntamente com outros indicadores para que a qualidade do solo seja avaliada.

Na camada superficial $(0,00-0,05 m)$, não foram observadas diferenças entre tratamentos para os parâmetros $\mathrm{D}_{\mathrm{S}}$ e $\mathrm{P}_{\mathrm{T}}$. Por outro lado, a $\mathrm{M}_{\mathrm{A}}$ revelou diferença entre os tratamentos $\mathrm{SD}_{\mathrm{ESC} 1}$ e $\mathrm{SD}_{\mathrm{COMP} 2}$ e $\mathrm{SD}_{\mathrm{ESC} 1}$ e SC. A $M_{I}$ foi significativa entre os tratamentos SD e $\mathrm{SD}_{\text {СомР3 }}, \mathrm{SD}_{\text {ESC1 }}$ e $\mathrm{SD}_{\text {СомР2 }}, \mathrm{SD}_{\mathrm{ESC1}}$ e $\mathrm{SD}_{\text {СомР3 }}, \mathrm{SD}_{\mathrm{ESC1}}$ e $\mathrm{SC}, \mathrm{SD}_{\text {СомР1 }}$ e $\mathrm{SD}_{\text {СомР3 }}$ e $\mathrm{SD}_{\text {СомР3 } 3}$ e $\mathrm{SD}_{\mathrm{ESC2}}$. O sistema de manejo que recebeu compactação adicional em dois anos agrícolas $\left(\mathrm{SD}_{\text {Сомез }}\right.$ ) apresentou diferenças significativas quando contrastado com outros tratamentos (Tabela 3).

Tabela 2 - Valores médios de condutividade hidráulica de solo saturado $\left(\mathrm{K}_{\theta \mathrm{S}}\right)$, densidade do solo $\left(\mathrm{D}_{\mathrm{S}}\right)$, porosidade total $\left(\mathrm{P}_{\mathrm{T}}\right)$, macro $\left(\mathrm{M}_{\mathrm{A}}\right)$ e microporosidade $\left(\mathrm{M}_{\mathrm{I}}\right)$ na camada de $0,00-0,35 \mathrm{~m}$ e rendimento das culturas nos diferentes tratamentos.

\begin{tabular}{|c|c|c|c|c|c|c|c|}
\hline Tratamentos $^{1}$ & $\mathrm{~K}_{\theta \mathrm{S}}, \mathrm{mm} \mathrm{h}^{-1}$ & $\mathrm{D}_{\mathrm{s}}, \mathrm{Mg} \mathrm{m}^{-3}$ & $\mathrm{P}_{\mathrm{T}}, \mathrm{m}^{3} \mathrm{~m}^{-3}$ & $\mathrm{M}_{\mathrm{A}}, \mathrm{m}^{3} \mathrm{~m}^{-3}$ & $\mathrm{M}_{\mathrm{I}}, \mathrm{m}^{3} \mathrm{~m}^{-3}$ & \multicolumn{2}{|c|}{ Rendimento, kg ha ${ }^{-1}$} \\
\hline & & & & & & Soja & Feijoeiro \\
\hline SD & 44,40 & 1,68 & 0,32 & 0,07 & 0,24 & 3139 & 1498 \\
\hline $\mathrm{SD}_{\mathrm{ESC} 1}$ & 14,62 & 1,71 & 0,32 & 0,07 & 0,25 & 2978 & 1848 \\
\hline $\mathrm{SD}_{\mathrm{ESC} 2}$ & 15,14 & 1,69 & 0,33 & 0,08 & 0,25 & - & 1395 \\
\hline $\mathrm{SD}_{\mathrm{COMP} 1}$ & 16,11 & 1,74 & 0,31 & 0,06 & 0,25 & - & 1251 \\
\hline $\mathrm{SD}_{\text {СOMP2 }}$ & 25,44 & 1,73 & 0,31 & 0,07 & 0,25 & - & 1488 \\
\hline $\mathrm{SD}_{\text {СOMP3 }}$ & 10,42 & 1,82 & 0,29 & 0,06 & 0,23 & 2898 & - \\
\hline SC & 28,22 & 1,66 & 0,35 & 0,10 & 0,25 & 2771 & 1596 \\
\hline
\end{tabular}

${ }^{1} \mathrm{SD}=$ sistema de semeadura direta desde o ano de 1989; $\mathrm{SD}_{\mathrm{ESC} 1}=$ sistema de semeadura direta escarificado em dezembro de $2004 ; \mathrm{SD}_{\mathrm{ESC} 2}=$ sistema de semeadura direta escarificado em dezembro de 2002 e fevereiro de $2004 ; \mathrm{SD}_{\mathrm{COMP} 1}=$ sistema de semeadura direta com 4 passadas

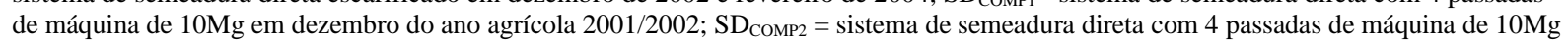
em dezembro do ano agrícola 2002/2003; $\mathrm{SD}_{\mathrm{COMP} 3}=$ sistema de semeadura direta com 4 passadas de máquina de $10 \mathrm{Mg}$ em dezembro do ano agrícola 2001/2002 e 2002/2003 e SC = sistema convencional de preparo do solo. 
Tabela 3 - Valor de $\mathrm{F}$ e contrastes significativos entre os tratamentos das variáveis: condutividade hidráulica de solo saturado (K $\mathrm{K}_{\theta \mathrm{S}}$ ), densidade do solo $\left(\mathrm{D}_{\mathrm{S}}\right)$, porosidade total, $\left(\mathrm{P}_{\mathrm{T}}\right)$, macroporosidade $\left(\mathrm{M}_{\mathrm{A}}\right)$ e microporosidade $\left(\mathrm{M}_{\mathrm{I}}\right)$ nas camadas de 0,00-0,05 e 0,10$0,15 \mathrm{~m}$.

\begin{tabular}{|c|c|c|c|c|c|}
\hline Contrastes $^{1}$ & $\mathrm{~K}_{\theta \mathrm{S}}$ & $\mathrm{D}_{\mathrm{S}}$ & $\mathrm{P}_{\mathrm{T}}$ & $\mathrm{M}_{\mathrm{A}}$ & $\mathrm{M}_{\mathrm{I}}$ \\
\hline \multicolumn{6}{|c|}{ Camada de $0,00-0,05 \mathrm{~m}$} \\
\hline $\mathrm{SD}$ e $\mathrm{SD}_{\text {СОМР3 }}$ & $1,05 \mathrm{~ns}$ & $1,49 \mathrm{~ns}$ & $3,15 \mathrm{~ns}$ & $0,00 \mathrm{~ns}$ & $11,27 * *$ \\
\hline $\mathrm{SD}_{\mathrm{ESC} 1}$ e $\mathrm{SD}_{\text {СомP2 }}$ & $0,51 \mathrm{~ns}$ & 0,45 ns & $0,60 \mathrm{~ns}$ & $4,68 *$ & $6,09 *$ \\
\hline $\mathrm{SD}_{\mathrm{ESC} 1}$ e $\mathrm{SD}_{\mathrm{COMP} 3}$ & $0,00 \mathrm{~ns}$ & $0,53 \mathrm{~ns}$ & $0,94 \mathrm{~ns}$ & $1,52 \mathrm{~ns}$ & $15,83 * *$ \\
\hline $\mathrm{SD}_{\mathrm{ESC} 1}$ e SC & $0,74 \mathrm{~ns}$ & 2,32 ns & $1,37 \mathrm{~ns}$ & $7,58 * *$ & $7,53 * *$ \\
\hline $\mathrm{SD}_{\text {СOMP1 }}$ e $\mathrm{SD}_{\text {СОMP3 }}$ & $0,08 \mathrm{~ns}$ & $0,36 \mathrm{~ns}$ & $2,00 \mathrm{~ns}$ & $0,04 \mathrm{~ns}$ & $5,01 *$ \\
\hline $\mathrm{SD}_{\text {Сомт3 }}$ e $\mathrm{SD}_{\mathrm{ESC} 2}$ & $0,01 \mathrm{~ns}$ & $0,32 \mathrm{~ns}$ & $1,56 \mathrm{~ns}$ & $0,13 \mathrm{~ns}$ & $8,61 * *$ \\
\hline \multicolumn{6}{|c|}{ Camada de $0,10-0,15 \mathrm{~m}$} \\
\hline $\mathrm{SD}$ e $\mathrm{SD}_{\text {СОMP3 }}$ & $0,87 \mathrm{~ns}$ & $9,44 * *$ & $0,53 \mathrm{~ns}$ & $0,36 \mathrm{~ns}$ & $0,32 \mathrm{~ns}$ \\
\hline $\mathrm{SD}$ e $\mathrm{SD}_{\text {СомP2 }}$ & 1,20 ns & 1,25 ns & $0,23 \mathrm{~ns}$ & 2,92 ns & $6,97 *$ \\
\hline SD e SC & $0,02 \mathrm{~ns}$ & $13,20 * *$ & $21,72 * *$ & $11,47 * *$ & $15,05 * *$ \\
\hline $\mathrm{SD}_{\mathrm{ESC} 1}$ e $\mathrm{SD}_{\mathrm{COMP} 3}$ & $0,00 \mathrm{~ns}$ & $4,78 *$ & $0,64 \mathrm{~ns}$ & $0,26 \mathrm{~ns}$ & $0,59 \mathrm{~ns}$ \\
\hline $\mathrm{SD}_{\mathrm{ESC} 1}$ e $\mathrm{SD}_{\mathrm{COMP} 2}$ & $0,02 \mathrm{~ns}$ & $0,12 \mathrm{~ns}$ & $0,11 \mathrm{~ns}$ & $2,42 \mathrm{~ns}$ & $5,07 *$ \\
\hline $\mathrm{SD}_{\mathrm{ESC} 1}$ e $\mathrm{SC}$ & $1,31 \mathrm{~ns}$ & $17,37 * *$ & $17,51 * *$ & $10,23 * *$ & $11,04 * *$ \\
\hline $\mathrm{SD}_{\text {СОмР3 }}$ e $\mathrm{SD}_{\mathrm{ESC} 2}$ & $0,11 \mathrm{~ns}$ & $9,11 * *$ & 2,86 ns & $1,31 \mathrm{~ns}$ & 2,39 ns \\
\hline $\mathrm{SD}_{\text {СОмР2 }}$ e $\mathrm{SD}_{\text {СОмР3 }}$ & $0,02 \mathrm{~ns}$ & $2,55 \mathrm{~ns}$ & $0,96 \mathrm{~ns}$ & $0,82 \mathrm{~ns}$ & $6,85 *$ \\
\hline $\mathrm{SD}_{\text {СомРз }}$ e $\mathrm{SC}$ & $0,87 \mathrm{~ns}$ & $30,94 * *$ & $18,15 * *$ & $10,00 * *$ & $12,32 * *$ \\
\hline $\mathrm{SD}_{\text {СOMP1 }}$ e $\mathrm{SC}$ & $0,97 \mathrm{~ns}$ & $14,39 * *$ & $13,65 * *$ & $10,00 * *$ & $6,52 *$ \\
\hline $\mathrm{SD}_{\mathrm{ESC} 2}$ e $\mathrm{SD}_{\mathrm{COMP} 2}$ & $0,22 \mathrm{~ns}$ & $2,02 \mathrm{~ns}$ & $0,50 \mathrm{~ns}$ & $4,20 *$ & 1,14 ns \\
\hline $\mathrm{SD}_{\mathrm{ESC} 2}$ e SC & $0,32 \mathrm{~ns}$ & $5,09 *$ & $5,80 *$ & $3,64 \mathrm{~ns}$ & $3,29 \mathrm{~ns}$ \\
\hline $\mathrm{SD}_{\mathrm{COMP} 2}$ e $\mathrm{SC}$ & $1,16 \mathrm{~ns}$ & $14,54 * *$ & $10,14 * *$ & $17,24 * *$ & $0,41 \mathrm{~ns}$ \\
\hline
\end{tabular}

${ }^{1} \mathrm{SD}=$ sistema de semeadura direta desde o ano de 1989; $\mathrm{SD}_{\mathrm{ESC} 1}=$ sistema de semeadura direta escarificado em dezembro de 2004 ; $\mathrm{SD}_{\mathrm{ESC} 2}=$ sistema de semeadura direta escarificado em dezembro de 2002 e fevereiro de 2004; SD $_{\text {COMP1 }}=$ sistema de semeadura direta com 4 passadas de máquina de $10 \mathrm{Mg}$ em dezembro do ano agrícola 2001/2002; $\mathrm{SD}_{\mathrm{COMP} 2}=$ sistema de semeadura direta com 4 passadas de máquina de 10 Mg em dezembro do ano agrícola 2002/2003; SD $_{\text {СомР3 }}=$ sistema de semeadura direta com 4 passadas de máquina de 10 Mg em dezembro do ano agrícola 2001/2002 e 2002/2003 e SC = sistema convencional de preparo do solo. ns = não significativo; ** significativo a $1 \%$; * significativo a $5 \%$.

Na camada de 0,10-0,15m, de maneira geral, foram observadas diferenças significativas para os parâmetros de $\mathrm{D}_{S}, \mathrm{P}_{\mathrm{T}}, \mathrm{M}_{\mathrm{A}}$ e $\mathrm{M}_{\mathrm{I}}$ entre os tratamentos SD e SC, $S_{\text {ESC1 }}$ e SC, SD ${ }_{\text {COMP1 }}$ e SC e SD ${ }_{\text {COMP3 }}$ e SC e, com exceção da $M_{I}$, diferenças foram obtidas entre $S_{\text {COMP2 }}$ e SC (Tabela 3). O sistema que recebeu aração e gradagem (SC) contrastou significativamente com praticamente todos os tratamentos devido à maior desestruturação causada pelo revolvimento do solo, possibilitando maiores diferenças na qualidade estrutural.

Foram observadas diferenças significativas de $D_{S}, P_{T}$ e $M_{A}$ na camada de 0,20-0,25m entre os tratamentos $\mathrm{SD}_{\mathrm{ESC2}}$ e SD $\mathrm{COMP} 3_{\text {COMP1 }}$ e SC, $\mathrm{SD}_{\mathrm{COMP} 2}$ e $\mathrm{SC}$ e $\mathrm{SD}_{\text {Сомp3 }}$ e SC. A $\mathrm{M}_{\mathrm{I}}$ nessa camada foi distinta entre os tratamentos SD e $\mathrm{SD}_{\mathrm{ESC} 2}, \mathrm{SD}_{\mathrm{ESC} 1}$ e $\mathrm{SD}_{\mathrm{COMP} 1}$, $\mathrm{SD}_{\mathrm{ESC1}}$ e $\mathrm{SD}_{\mathrm{ESC2}}, \mathrm{SD}_{\mathrm{ESC1}}$ e SC, SD $\mathrm{COMP}_{\text {COM }}$ e SD e $\mathrm{SD}_{\mathrm{ESC} 2}$ (Tabela 4). Novamente, os tratamentos que receberam maior revolvimento ( $S_{E S C 1}$ e $S C$ ) possibilitaram maiores diferenças estruturais do solo em relação aos demais.

Na camada de 0,30-0,35m, os contrastes entre os tratamentos SD e $\mathrm{SD}_{\text {СомР2 }}$, SD e $\mathrm{SD}_{\text {СомР3 }}$ e
$\mathrm{SD}_{\mathrm{ESC1}}$ e $\mathrm{SD}_{\mathrm{COMP} 3}$ apresentaram diferença para as variáveis $D_{S}$ e $M_{A}$. Analisando-se somente os contrastes ortogonais da $\mathrm{D}_{\mathrm{s}}$, diferenças foram obtidas entre SD e SC, $\mathrm{SD}_{\text {СомР1 }}$ e $\mathrm{SD}_{\text {СОмР3 }}$, $\mathrm{SD}_{\text {СомР3 }}$ e $\mathrm{SD}_{\mathrm{ESC} 2}$ e $\mathrm{SD}_{\mathrm{ESC} 2}$ e SC. Os contrastes ortogonais da $\mathrm{M}_{\mathrm{I}}$ e $\mathrm{D}_{\mathrm{S}}$ mostraram significância somente entre SD e SC.A porosidade total não revelou diferençaS entre os tratamentos (Tabela 4).

Dentre os contrastes ortogonais e variáveis significativas, observou-se que os tratamentos $\mathrm{SD}_{\text {СомРз }}$ e SC, ou seja, o maior e menor nível de compactação, foram os que, de modo geral, contrastaram significativamente com os demais. O sistema convencional de preparo e o sistema de semeadura direta, que recebeu compactação adicional em dois anos agrícolas ( $\mathrm{SD}_{\text {СомР3 }}$ ), apresentaram diferenças notáveis com relação à qualidade estrutural do solo. De acordo com SILVA et al. (1994), dentre as etapas do manejo, o preparo do solo talvez seja a atividade que mais influencia o comportamento físico do solo, pois atua diretamente na estrutura, podendo causar modificações na porosidade, na retenção de água e na resistência 
Tabela 4 - Valor de F e contrastes significativos entre os tratamentos das variáveis: condutividade hidráulica de solo saturado (K $\mathrm{K}_{\theta \mathrm{S}}$ ), densidade do solo $\left(\mathrm{D}_{\mathrm{S}}\right)$, porosidade total, $\left(\mathrm{P}_{\mathrm{T}}\right)$, macroporosidade $\left(\mathrm{M}_{\mathrm{A}}\right)$ e microporosidade $\left(\mathrm{M}_{\mathrm{I}}\right)$ nas camadas de 0,20-0,25 e 0,30$0,35 \mathrm{~m}$.

\begin{tabular}{|c|c|c|c|c|c|}
\hline Contrastes $^{1}$ & $\mathrm{~K}_{\theta \mathrm{S}}$ & $\mathrm{D}_{\mathrm{S}}$ & $\mathrm{P}_{\mathrm{T}}$ & $\mathrm{M}_{\mathrm{A}}$ & $\mathrm{M}_{\mathrm{I}}$ \\
\hline \multicolumn{6}{|c|}{ Camada de $0,20-0,25 \mathrm{~m}$} \\
\hline $\mathrm{SD}$ e $\mathrm{SD}_{\text {СOMP1 }}$ & $1,22 \mathrm{~ns}$ & $6,80 *$ & $0,95 \mathrm{~ns}$ & $4,99 *$ & $4,06 \mathrm{~ns}$ \\
\hline $\mathrm{SD}$ e $\mathrm{SD}_{\text {СОмР3 }}$ & $1,35 \mathrm{~ns}$ & $21,02 * *$ & $4,09 \mathrm{~ns}$ & $4,14 *$ & $0,29 \mathrm{~ns}$ \\
\hline $\mathrm{SD}$ e $\mathrm{SD}_{\mathrm{ESC2}}$ & $0,87 \mathrm{~ns}$ & $0,04 \mathrm{~ns}$ & $1,79 \mathrm{~ns}$ & $0,09 \mathrm{~ns}$ & $4,62 *$ \\
\hline SD e SC & $0,98 \mathrm{~ns}$ & $0,70 \mathrm{~ns}$ & $10,52 * *$ & $7,88 * *$ & $2,46 \mathrm{~ns}$ \\
\hline $\mathrm{SD}_{\mathrm{ESC} 1}$ e $\mathrm{SD}_{\mathrm{COMP} 1}$ & $0,37 \mathrm{~ns}$ & $8,38 * *$ & 1,93 ns & $9,61 * *$ & $7,41 *$ \\
\hline $\mathrm{SD}_{\mathrm{ESC} 2}$ e $\mathrm{SD}_{\mathrm{COMP} 3}$ & $0,44 \mathrm{~ns}$ & $22,65 * *$ & $5,65 *$ & $8,48 * *$ & $0,10 \mathrm{~ns}$ \\
\hline $\mathrm{SD}_{\mathrm{ESC} 1}$ e $\mathrm{SD}_{\mathrm{ESC} 2}$ & $0,20 \mathrm{~ns}$ & $0,40 \mathrm{~ns}$ & $0,63 \mathrm{~ns}$ & $0,51 \mathrm{~ns}$ & $8,11 * *$ \\
\hline $\mathrm{SD}_{\mathrm{ESC} 1}$ e $\mathrm{SD}_{\mathrm{COMP} 2}$ & $0,01 \mathrm{~ns}$ & $3,86 \mathrm{~ns}$ & 3,02 ns & $5,86 *$ & $0,55 \mathrm{~ns}$ \\
\hline $\mathrm{SD}_{\mathrm{ESC} 1}$ e SC & $0,18 \mathrm{~ns}$ & $0,08 \mathrm{~ns}$ & $6,10 *$ & $2,17 \mathrm{~ns}$ & $5,68 *$ \\
\hline $\mathrm{SD}_{\mathrm{COMP} 1}$ e $\mathrm{SD}_{\text {СOMP3 }}$ & $0,00 \mathrm{~ns}$ & $2,61 \mathrm{~ns}$ & $0,73 \mathrm{~ns}$ & $0,03 \mathrm{~ns}$ & $4,34 *$ \\
\hline $\mathrm{SD}_{\mathrm{COMP} 3}$ e $\mathrm{SD}_{\mathrm{ESC} 2}$ & $0,04 \mathrm{~ns}$ & $12,77 * *$ & $7,53 * *$ & 3,63 ns & $4,81 *$ \\
\hline $\mathrm{SD}_{\text {СОмР2 }}$ e $\mathrm{SD}_{\text {СомР3 }}$ & $0,46 \mathrm{~ns}$ & $5,86 *$ & $0,31 \mathrm{~ns}$ & $0,18 \mathrm{~ns}$ & $0,13 \mathrm{~ns}$ \\
\hline $\mathrm{SD}_{\mathrm{COMP} 3}$ e $\mathrm{SC}$ & $0,08 \mathrm{~ns}$ & $22,54 * *$ & $18,67 * *$ & $15,96 * *$ & $2,87 \mathrm{~ns}$ \\
\hline $\mathrm{SD}_{\text {COMP1 }}$ e $\mathrm{SD}_{\mathrm{ESC} 2}$ & $0,02 \mathrm{~ns}$ & $3,84 \mathrm{~ns}$ & $3,57 \mathrm{~ns}$ & $4,27 *$ & $0,01 \mathrm{~ns}$ \\
\hline $\mathrm{SD}_{\text {СOMP1 }}$ e SC & $0,05 \mathrm{~ns}$ & $8,88 * *$ & $11,45 * *$ & $17,41 * *$ & $0,34 \mathrm{~ns}$ \\
\hline $\mathrm{SD}_{\mathrm{ESC} 2}$ e $\mathrm{SD}_{\mathrm{COMP} 2}$ & $0,24 \mathrm{~ns}$ & 1,33 ns & $4,81 *$ & $2,19 \mathrm{~ns}$ & $3,34 \mathrm{~ns}$ \\
\hline $\mathrm{SD}_{\mathrm{COMP} 2}$ e SC & $0,22 \mathrm{~ns}$ & $4,39 *$ & $13,81 *$ & $12,45 * *$ & $1,68 \mathrm{~ns}$ \\
\hline \multicolumn{6}{|c|}{ Camada de $0,30-0,35 \mathrm{~m}$} \\
\hline SD e $\mathrm{SD}_{\text {СОMP2 }}$ & $1,37 \mathrm{~ns}$ & $4,99 *$ & 2,91 ns & $4,65 *$ & $0,08 \mathrm{~ns}$ \\
\hline $\mathrm{SD}$ e $\mathrm{SD}_{\text {СОМР3 }}$ & $1,32 \mathrm{~ns}$ & $18,29 * *$ & $4,10 \mathrm{~ns}$ & $4,32 *$ & $0,25 \mathrm{~ns}$ \\
\hline SD e SC & $1,86 \mathrm{~ns}$ & $16,78 * *$ & $0,00 \mathrm{~ns}$ & $1,44 \mathrm{~ns}$ & $3,65 \mathrm{~ns}$ \\
\hline $\mathrm{SD}_{\mathrm{ESC} 1}$ e $\mathrm{SD}_{\mathrm{COMP} 2}$ & $0,08 \mathrm{~ns}$ & $0,78 \mathrm{~ns}$ & $2,69 \mathrm{~ns}$ & $4,94 *$ & $0,30 \mathrm{~ns}$ \\
\hline $\mathrm{SD}_{\mathrm{ESC} 1}$ e $\mathrm{SD}_{\mathrm{COMP} 3}$ & $0,07 \mathrm{~ns}$ & $7,90 * *$ & $3,77 \mathrm{~ns}$ & $4,62 *$ & $0,04 \mathrm{~ns}$ \\
\hline $\mathrm{SD}_{\mathrm{ESC} 1}$ e SC & $0,11 \mathrm{~ns}$ & $5,80 *$ & $0,00 \mathrm{~ns}$ & $1,76 \mathrm{~ns}$ & $4,31 *$ \\
\hline $\mathrm{SD}_{\text {СOMP1 }}$ e $\mathrm{SD}_{\mathrm{COMP} 3}$ & $0,00 \mathrm{~ns}$ & $4,22 *$ & $0,93 \mathrm{~ns}$ & $0,17 \mathrm{~ns}$ & $1,48 \mathrm{~ns}$ \\
\hline $\mathrm{SD}_{\mathrm{COMP} 3}$ e $\mathrm{SD}_{\mathrm{ESC} 2}$ & $0,00 \mathrm{~ns}$ & $8,72 * *$ & 3,73 ns & $2,31 \mathrm{~ns}$ & $1,48 \mathrm{~ns}$ \\
\hline $\mathrm{SD}_{\mathrm{ESC} 2}$ e SC & $0,01 \mathrm{~ns}$ & $6,67 *$ & $0,09 \mathrm{~ns}$ & $0,54 \mathrm{~ns}$ & $0,35 \mathrm{~ns}$ \\
\hline
\end{tabular}

${ }^{1} \mathrm{SD}=$ sistema de semeadura direta desde o ano de 1989; $\mathrm{SD}_{\mathrm{ESC} 1}=$ sistema de semeadura direta escarificado em dezembro de 2004 ; $\mathrm{SD}_{\mathrm{ESC} 2}=$ sistema de semeadura direta escarificado em dezembro de 2002 e fevereiro de 2004; $\mathrm{SD}_{\mathrm{COMP} 1}=$ sistema de semeadura direta com 4 passadas de máquina de 10Mg em dezembro do ano agrícola 2001/2002; $\mathrm{SD}_{\mathrm{COMP} 2}=$ sistema de semeadura direta com 4 passadas de máquina de $10 \mathrm{Mg}$ em dezembro do ano agrícola 2002/2003; $\mathrm{SD}_{\mathrm{COMP} 3}=$ sistema de semeadura direta com 4 passadas de máquina de $10 \mathrm{Mg}$ em dezembro do ano agrícola 2001/2002 e 2002/2003 e SC = sistema convencional de preparo do solo. ns = não significativo; ** significativo a 1\%; * significativo a $5 \%$.

mecânica à penetração. SILVA et al. (2003) observaram que a intensidade do tráfego do rodado das máquinas agrícolas pode alterar a densidade, a porosidade e a condutividade hidráulica do solo saturado na profundidade superficial $(0,00-0,05 \mathrm{~m})$, na profundidade média de trabalho $(0,24-0,27 \mathrm{~m})$ e na profundidade de corte de implementos como arado de discos, arado de aivecas e grade aradora.

Quanto à faixa de umidade volumétrica ótima durante o ciclo do feijoeiro, observou-se que o SD apresentou maior período dentro dessa faixa (Figura 1). De modo geral, a faixa mais estreita de umidade ótima foi observada no sistema que recebeu compactação adicional, corroborando os resultados de COLLARES (2005). Estudos têm evidenciado que a água do solo pode ser incrementada em sistema semeadura direta pelo efeito da cobertura vegetal na redução da evaporação e no aumento da armazenagem de água (STONE \& SILVEIRA, 1999).

A maior produtividade média da soja e do feijoeiro foi observada, respectivamente, no SD e no $\mathrm{SD}_{\mathrm{ESC} 1}$ (Tabela 2). Foi verificada, a partir dos contrastes ortogonais, diferença de rendimento somente para a cultura do feijoeiro entre os tratamentos $\mathrm{SD}_{\mathrm{ESC1}}(2.978 \mathrm{~kg}$ $\left.\mathrm{ha}^{-1}\right)$ e $\mathrm{SD}_{\text {COMP1 }}\left(1.251 \mathrm{~kg} \mathrm{ha}^{-1}\right)$ e entre $\mathrm{SD}_{\text {ESC1 }}$ e SD $\mathrm{ESC}_{\mathrm{C}}$ $\left(1.395 \mathrm{~kg} \mathrm{ha}^{-1}\right)$. O estresse hídrico na fase vegetativa pode ter influenciado a produtividade do feijoeiro. A escarificação na semeadura ( $\mathrm{SD}_{\mathrm{ESC} 1}$ ) possibilitou maior rendimento do feijoeiro, porém esse efeito evidenciouse no primeiro ano, pois o tratamento com escarificação 


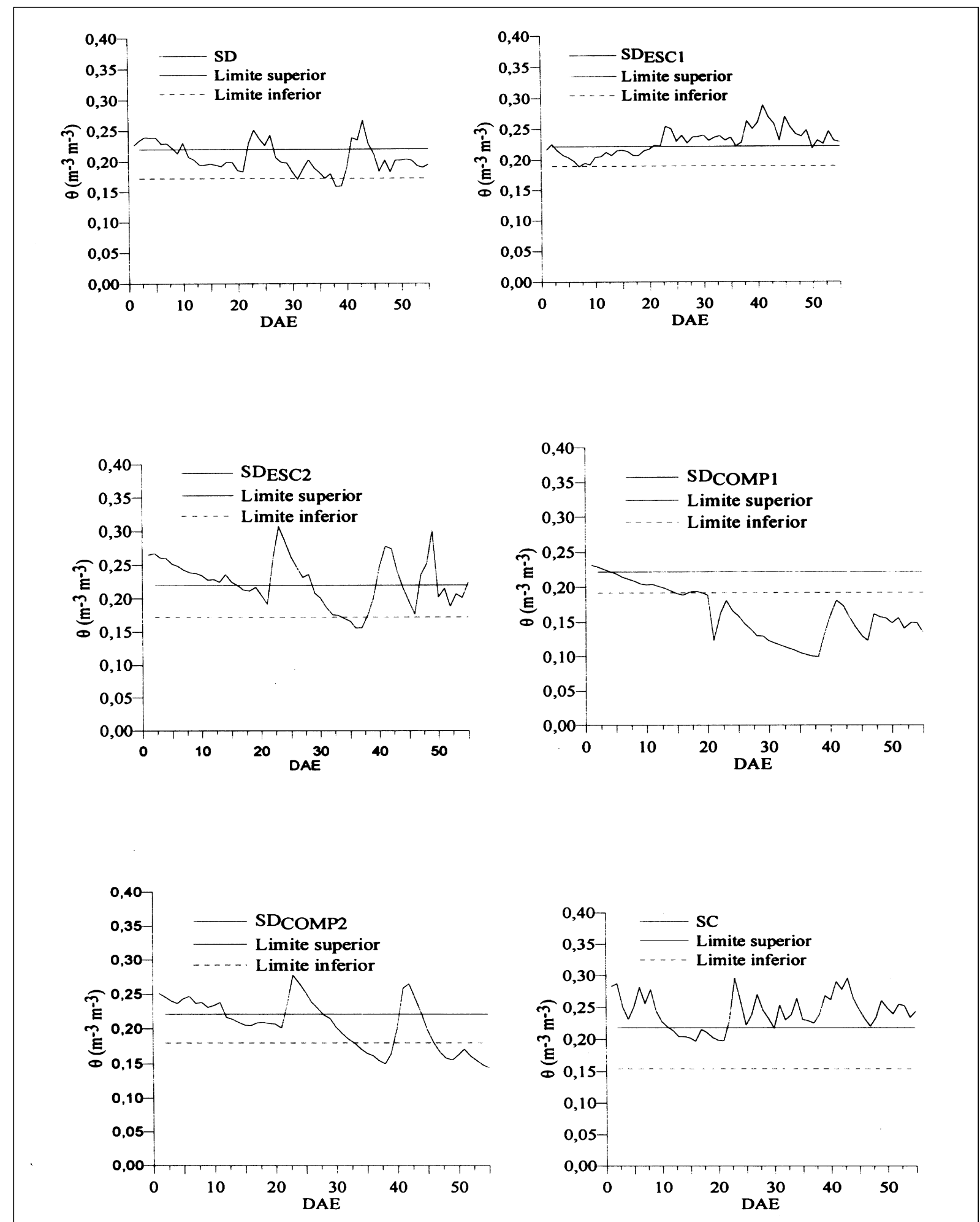

Figura 1 - Limite superior e inferior de umidade volumétrica $(\theta)$ ótima para um Argissolo Vermelho distrófico na camada de 0,00$0,20 \mathrm{~m}$, nos diferentes tratamentos ${ }^{1} .{ }^{1} \mathrm{SD}=$ sistema de semeadura direta desde o ano de $1989 ; \mathrm{SD}_{\mathrm{ESC1}}=$ sistema de semeadura direta escarificado em dezembro de 2004; $\mathrm{SD}_{\mathrm{ESC} 2}=$ sistema de semeadura direta escarificado em dezembro de 2002 e fevereiro de 2004; $\mathrm{SD}_{\mathrm{COMP1}}=$ sistema de semeadura direta com 4 passadas de máquina de 10mg em dezembro do ano agrícola 2001/2002; $\mathrm{SD}_{\mathrm{COMP2}}=$ sistema de semeadura direta com 4 passadas de máquina de 10Mg em dezembro do ano agrícola 2002/2003 e SC = sistema convencional de preparo do solo.

Ciência Rural, v.36, n.4, jul-ago, 2006. 
em dezembro de 2002 e fevereiro de $2004\left(\mathrm{SD}_{\mathrm{ESC} 2}\right)$ apresentou um dos menores rendimentos, o que sugere uma rápida reacomodação das partículas desse solo.

\section{CONCLUSÕES}

As maiores alterações na qualidade físicohídrica foram observadas no sistema convencional de preparo e no sistema semeadura direta, que recebeu compactação adicional em dois anos agrícolas $\left(\mathrm{SD}_{\text {сомр3}}\right)$. O SD apresentou as melhores condições físico-hídricas e permaneceu por maior período dentro da faixa de umidade volumétrica considerada ótima para o desenvolvimento das culturas. A condutividade hidráulica de solo saturado não apresentou diferença entre os tratamentos. O rendimento da soja não foi influenciado pelos tratamentos, enquanto que a escarificação ( $\mathrm{SD}_{\mathrm{ESC1}}$ ) foi favorável ao rendimento do feijoeiro.

\section{AGRADECIMENTOS}

À Fundação de Amparo à Pesquisa do Rio Grande do Sul (FAPERGS), ao Conselho Nacional de Desenvolvimento Científico e Tecnológico (CNPq) e à Coordenação de Aperfeiçoamento de Pessoal de Nível Superior (CAPES), pela concessão de bolsas e pelo financiamento parcial.

\section{REFERÊNCIAS}

BLAKE, G.R.; HARTGE, K.H. Bulk density. In: KLUTE, A. Methods of soil analysis: physical and mineralogical methods. 2.ed. Madison: American Society of Agronomy, Soil Science Society of America, 1986. p.363-375.

BOONE, F.R.; VEEN, B.W. Mechanisms of crop responses to soil compaction. In: SOANE, B.D.; van OUWERKWRK, C. Soil compaction in crop production. Amsterdam: Elsevier, 1994. p.237-264.

COLLARES, G.L. Compactação em Latossolos e Argissolo e relação com parâmetros de solo e de plantas. 2005. 106f. Tese (Doutorado em Ciência do Solo) - Curso de Pósgraduação em solos, Universidade Federal de Santa Maria.

COQUET, Y. et al. Vertical variation of near saturated hydraulic conductivity in three soil profiles. Geoderma, v.126, p.181191, 2005.

DALMOLIN, R.S.D. et al. Correspondência entre o sistema brasileiro de classificação de solos (EMBRAPA, 1999), WRB (ISSSWG, 1998) e o Soil Taxonomy (USDA, 1999) para algumas unidade de solos do Rio Grande do Sul. Santa Maria: Centro de Ciências Rurais, UFSM, 2004. 4p. (Informe Técnico, 10).

DEFOSSEZ, P.; RICHARD, G. Models of soil compaction due to traffic and their evaluation. Soil and Tillage Research, v.67, p.41-64, 2002.
EMBRAPA. Centro Nacional de Pesquisa de Solos, Rio de Janeiro, RJ. Manual de métodos de análise de solo. 2.ed. Rio de Janeiro: Embrapa - Solos, 1997. 212p.

EMBRAPA. Centro Nacional de Pesquisa de Solos. Sistema brasileiro de classificação de solos. Brasília: Embrapa Produção de Informação, Rio de Janeiro: Embrapa: Solos, 1999. 412p.

FLOWERS, M.D.; LAL, R. Axle load and tillage effects on soil physical properties and soybean grain yield on a mollic ochraqualf in northwest Ohio. Soil and Tillage Research, v.48, p.21-35, 1998.

GUROVICH, L.A. Field spatial variability structure of soil hydrodynamic properties. Ciência e Investigación Agraria, v.9, p.243-254, 1982.

HORN, R. et al. Effect of repeated tractor wheeling on stress/ strain properties and consequences on physical properties in structured arable soils. Soil and Tillage Research, v.73, p.101-106, 2003.

KARLEN, D.L. et al. Soil quality: Why and how? Geoderma, v.114, p.145-156, 2003.

KOPPI, A.J.; DOUGLAS, J.T. A rapid inexpensive and quantitative procedure for assessing soil structure with respect to cropping. Soil Use Management, v.7, p.52$56,1991$.

LAL, R. Soil compaction and tillage effects on soil physical properties of a mollic ochraqualf in northwest Ohio. Journal of Sustainable Agriculture, v.14, n.4, p.53-65, 1999.

LHOTSKÝ, J. et al. Degradation of soil by increasing compression. Soil and Tillage Research, v.19, p.287-295, 1991.

OSUNBITAN, J.A. et al. Tillage effects on bulk density, hydraulic conductivity and strength of a loamy sandy soil in southwestern Nigeria. Soil and Tillage Research, v.82, p.5764, 2005

SECCO, D. et al. Produtividade de soja e propriedades físicas de um Latossolo submetido a sistemas de manejo e compactação. Revista Brasileira de Ciência do Solo, v.28, p.797-804, 2004.

SILVA, A.P. et al. Characterization of the least limiting water range of soils. Soil Science Society of America Journal, v.58, p.1775-1781, 1994.

SILVA, R.B. et al. O tráfego de máquinas agrícolas e as propriedades físicas, hídricas e mecânicas de um Latossolo dos cerrados. Revista Brasileira de Ciência do Solo, v.27, p.973-983, 2003.

SILVA, V.R. et al. Susceptibilidade à compactação de um Latossolo Vermelho Escuro e de um Podzólico Vermelho Amarelo. Revista Brasileira de Ciência do Solo, v.24, n.2, p.239-249, 2000.

STONE, L.F.; SILVEIRA, P.M. da. Efeitos do sistema de preparo na compactação do solo, disponibilidade hídrica e comportamento do feijoeiro. Pesquisa Agropecuária Brasileira, v.34, p.83-91, 1999.

WARRICK, A.; NIELSEN, D.R. Spatial variability of soil physical properties in the field. In: HILLEL, D. Applications of soil physics. New York: Academic, 1980. p.319-344.

WU, L. et al. Soil management effects on the nonlimiting water range. Geoderma, v.114, p.401-414, 2003. 